Sharif University of Technology
Scientia Iranica
SCIENTIA
IRAN I CA

\title{
Soret and Dufour effects on viscoelastic boundary layer flow over a stretching surface with convective boundary condition with radiation and chemical reaction
}

\author{
S. Eswaramoorthi ${ }^{a}$, M. Bhuvaneswari ${ }^{b}$, S. Sivasankaran $^{\mathrm{b}, *}$ and S. Rajan ${ }^{\mathrm{c}}$ \\ a. Department of Mathematics, Dr. N.G.P. Arts \& Science College, Coimbatore 641048, Tamil Nadu, India. \\ b. Institute of Mathematical Sciences, University of Malaya, Kuala Lumpur 50603, Malaysia. \\ c. Department of Mathematics, Erode Arts \& Science College, Erode 638009, Tamil Nadu, India. \\ Received 8 October 2014; received in revised form 26 July 2015; accepted 28 December 2015
}

\author{
KEYWORDS \\ Heat/mass transfer; \\ Viscoelastic fluid; \\ Homotopy analysis \\ method; \\ Radiation; \\ Soret/Dufour effects; \\ Chemical reaction.
}

\begin{abstract}
In this article, we investigate the double diffusive flow of a viscoelastic fluid on a stretching paper with convective boundary condition under the influence of thermal-diffusion and diffusion-thermo effects, thermal radiation, internal heat generation or absorption, chemical reaction, and thermal radiation. The governing boundary layer equations are analytically solved by using Homotopy Analysis Method (HAM). Variations of the velocity, concentration, and temperature profiles for different values of physical parameters are graphically displayed and discussed. Numerical results of the local Sherwood number and the local Nusselt number are also tabulated. It is observed that the local Nusselt number increases on increasing the radiation parameter. The local Sherwood number increases on increasing the chemical reaction parameter.
\end{abstract}

(C) 2016 Sharif University of Technology. All rights reserved.

\section{Introduction}

The study of viscoelastic boundary layer flow over a stretching surface has attracted considerable attention in the past few years due to many practical applications in industry and technology, e.g. polymer sheet extrusion from a dye, glass fiber, drawing of plastic films, and paper production. The manufacturing processes at high temperature need cooling and the flow may need viscosity to produce a good effect or to reduce the temperature. Heat and mass transfer of a viscoelastic fluid past a stretching vertical sheet was numerically studied by Hayat et al. [1]. They found that the velocity of the fluid and thickness of its boundary layer increased on increasing the viscoelastic parameter. MHD free convection flow and mass transfer of a viscoelastic fluid on a stretching surface were analytically studied by

\footnotetext{
*. Corresponding author.

E-mail address: sd.siva@yahoo.com (S. Sivasankaran)
}

Chowdhury and Islam [2]. Hayat et al. [3] analyzed the mass transfer of a viscoelastic fluid. The results of the analytical and numerical solutions were compared and established to be in excellent conformations. The mixed convection of a viscoelastic fluid flow over an isothermal flat spherical tube was numerically investigated by Anwar et al. [4]. It was found that the temperature and thermal boundary layer thickness increased when increasing the value of viscoelastic parameter. The effects of variable viscosity and heat transfer of a viscoelastic fluid in a porous medium were numerically studied by Chin et al. [5]. The heat and mass transfer of a viscoelastic fluid was analyzed by Sanjayanand and Khan [6]. It was found that increase in viscoelastic parameter led to the decrease in the local Skin friction.

Combined heat and mass transfer under the influence of radiation and chemical reaction arise in chemical industry for drying, chemical vapor depositions on surface, etc. Combined heat and mass transfer 
under the influence of first-order chemical ration by the side of a semi-infinite perpendicular surface was analyzed by Bhuvaneswari et al. [7]. They found that thickness of the thermal and concentration boundary layers decreased with increasing the chemical reaction parameter. Pal and Talukdar [8] investigated the MHD flow and mixed convective heat and mass transfer along a semi-infinite vertical plate with chemical reaction, Ohmic heating, and viscous dissipation. It was found that the velocity, as well as concentration boundary layer thickness, decreased on increasing the chemical reaction and thermal radiation parameters. Analytical solution of unsteady axisymmetric stagnation-point flow of a viscous fluid was investigated by Mozayyeni and Rahimi [9].

Free convective mass transfer of a Newtonian fluid in the presence of chemical reaction and magnetic field was analytically studied by Afify [10]. He found that the velocity, temperature, and concentration boundary layers decreased on increasing the chemical reaction parameter. Natural convective heat and mass transfer of a Newtonian fluid over a stretching surface was analyzed by Aboeldahab and Azzam [11]. Combined heat and mass transfer of a Newtonian fluid over a moving vertical flat plate with convective boundary condition was examined by Hamad et al. [12]. Hossain et al. [13] investigated the natural convective heat transfer flow with thermal radiation. Lee et al. [14] studied the natural convection heat transfer along an inclined plate in a porous medium. It was found that the thermal and momentum boundary layer thicknesses increased on increasing the radiation parameter. Bhuvaneswari et al. [15] examined the heat transfer and fluid flow by natural convection of an incompressible viscous fluid along a semi-infinite inclined plate in a fluid-saturated porous medium. They found that both velocity and temperature increased significantly when the value of the heat generation parameter increased. The problem of convection heat and mass transfer of an electrically conducting incompressible fluid over an inclined vertical plate in a porous medium under the influence of radiation and heat generation was analytically solved by Bhuvaneswari et al. [16]. It was observed that the temperature increased with increasing the values of the heat generation parameter. The heat transfer of a nanofluid over a stretching tube in the presence of heat source/sink was numerically investigated by Ahmed et al. [17]. They found that the local Nusselt number increased on increasing the Reynolds number.

In recent years, a large number of studies dealing with the effects of Soret and Dufour on heat and mass transfer problems of viscoelastic fluid have appeared. These properties are considered in secondorder phenomena and neglected in heat and mass transfer processes because they contain lesser orders of amount than the effects described by Fouriers and
Ficks laws. The effects of chemical reaction, and Soret and Dufour numbers on double diflusive convective flow in a porous medium were numerically studied by Postelnicu [18]. Hayat et al. [19] examined the Soret and Dufour effects on three-dimensional boundary layer flow of a viscoelastic fluid on a stretching surface. It was found that the behaviors of Soret number and Dufour number on temperature were quite opposite. Chamkha and Nakhi [20] analyzed the influence of thermal radiation, magnetic field, and Dufour and Soret effects on mixed convection heat and mass transfer from a vertical permeable plate embedded in a porous medium. They observed that both local Nusselt and Sherwood numbers decreased on increasing the Dufour number. MHD double diffusive natural convection from a vertical surface by taking an account of Soret and Dufour numbers was investigated by Postelnicu [21]. Soret and Dufour effects on double diffusive free convection from a vertical flat surface were studied by Rathish Kumar and Krishna Murthy [22].

The study of boundary layer flow with convective boundary condition is very important in different physical problems, such as transpiration cooling process and material drying. Several attractive computational studies of convective boundary condition have appeared in the recent past years [23-27]. It is noticed that the earlier literature on heat and mass transfer has mostly focused on either the viscoelastic fluid or viscous fluid with convective boundary condition. Hence, the main objective of our investigation is to study the double diffusive viscoelastic boundary layer flow with convective boundary condition. Analytical solutions for the velocity, concentration, and temperature profiles are developed by the homotopy analysis method [28]. This technique has been effectively applied by numerous researchers to various interesting problems.

\section{Mathematical formulation}

Consider the unsteady three-dimensional boundary layer flow of a viscoelastic fluid with convective boundary condition over a stretching paper at $z=0$. The $x$ - and $y$-axes are chosen in the plane of the surface and the $z$-axis is taken normal to it. The surrounding stationary fluid has concentration, $C_{\infty}$, and temperature, $T_{\infty}$, which is suficiently small in comparison to constant concentration, $C_{w}$, and temperature, $T_{w}$, at the surface. Soret and Dufour effects are included to study the heat and mass transfer. There is a first-order chemical reaction among the diffusing species and the fluid. The problem under consideration is governed by the following boundary layer equations:

$$
\frac{\partial u}{\partial x}+\frac{\partial v}{\partial y}+\frac{\partial w}{\partial z}=0
$$




$$
\begin{aligned}
& \frac{\partial u}{\partial t}+u \frac{\partial u}{\partial x}+v \frac{\partial u}{\partial y}+w \frac{\partial u}{\partial z}=\nu \frac{\partial^{2} u}{\partial z^{2}} \\
& -k_{0}\left(\frac{\partial^{3} u}{\partial z^{2} \partial t}+u \frac{\partial^{3} u}{\partial x \partial z^{2}}+w \frac{\partial^{3} u}{\partial z^{3}}-\frac{\partial u}{\partial x} \frac{\partial^{2} u}{\partial z^{2}}\right. \\
& \left.-\frac{\partial u}{\partial z} \frac{\partial^{2} w}{\partial z^{2}}-2 \frac{\partial u}{\partial z} \frac{\partial^{2} u}{\partial x \partial z}-2 \frac{\partial w}{\partial z} \frac{\partial^{2} u}{\partial z^{2}}\right), \\
& \frac{\partial v}{\partial t}+u \frac{\partial v}{\partial x}+v \frac{\partial v}{\partial y}+w \frac{\partial v}{\partial z}=\nu \frac{\partial^{2} v}{\partial z^{2}} \\
& -k_{0}\left(\frac{\partial^{3} v}{\partial z^{2} \partial t}+v \frac{\partial^{3} v}{\partial y \partial z^{2}}+w \frac{\partial^{3} v}{\partial z^{3}}-\frac{\partial v}{\partial y} \frac{\partial^{2} v}{\partial z^{2}}\right. \\
& \left.-\frac{\partial v}{\partial z} \frac{\partial^{2} w}{\partial z^{2}}-2 \frac{\partial v}{\partial z} \frac{\partial^{2} v}{\partial y \partial z}-2 \frac{\partial w}{\partial z} \frac{\partial^{2} v}{\partial z^{2}}\right), \\
& \frac{\partial C}{\partial t}+u \frac{\partial C}{\partial x}+v \frac{\partial C}{\partial y}+w \frac{\partial C}{\partial z}=D \frac{\partial^{2} C}{\partial z^{2}} \\
& -k_{1}\left(C-C_{\infty}\right)+\frac{D k_{T}}{T_{m}} \frac{\partial^{2} T}{\partial z^{2}}, \\
& \frac{\partial T}{\partial t}+u \frac{\partial T}{\partial x}+v \frac{\partial T}{\partial y}+w \frac{\partial T}{\partial z}=\alpha \frac{\partial^{2} T}{\partial z^{2}} \\
& +\frac{Q}{\rho c_{p}}\left(T-T_{\infty}\right)+\frac{D k_{T}}{c_{s} c_{p}} \frac{\partial^{2} C}{\partial z^{2}}-\frac{\alpha}{k_{T}} \frac{\partial q_{r}}{\partial z},
\end{aligned}
$$

where $u, v$, and $w$ are the velocities in the $x, y$, and $z$ directions, respectively. $\nu$ is the kinematic viscosity, $k_{0}$ is the material fluid parameter, $D$ is the diffusion coefficient, $k_{1}$ is the chemical reaction rate, $\alpha$ is the thermal diffusivity, $Q$ is the internal heat generation/absorption, $T_{m}$ is mean temperature of the fluid, $\rho$ is the density, $k_{T}$ is the thermal conductivity, $c_{s}$ is the concentration susceptibility, and $c_{p}$ is the specific heat.

With Rosseland approximation, the radiative heat flux is taken as:

$$
q_{r}=-\frac{4 \sigma_{0}}{3 k^{*}} \frac{\partial T^{4}}{\partial z}
$$

where $\sigma_{0}$ and $k^{*}$ are the Stefan-Boltzmann constant and mean absorption coefficient, respectively. The difference between the fluid temperature, $T$, and free stream temperature, $T_{\infty}$, is adequately small; thus, we neglect this difference. Using Taylor series, $T^{4}$ is expanded in terms of $T_{\infty}$; thus, $T^{4} \cong 4 T_{\infty}^{3} T-3 T_{\infty}^{4}$.

The boundary conditions are expressed as:

$$
\begin{aligned}
\text { At } z & =0: \\
\qquad & =u_{w}(x)=\frac{a x}{1-\alpha t}, \quad v=v_{w}(y)=\frac{b y}{1-\alpha t}, \\
w & =0, \quad C=C_{w}, \quad-k_{T} \frac{\partial T}{\partial z}=h\left(T_{w}-T\right),
\end{aligned}
$$

As $z \rightarrow \infty$ :

$$
u \rightarrow 0, \quad v \rightarrow 0, \quad C \rightarrow C_{\infty}, \quad T \rightarrow T_{\infty}
$$

where $a$ and $b$ are positive constants. Defining:

$$
\begin{aligned}
\eta & =\sqrt{\frac{a}{\nu(1-\alpha t)} z,} & u & =\frac{a x}{1-\alpha t} f^{\prime}(\eta) \\
v & =\frac{a y}{1-\alpha t} g^{\prime}(\eta), & w & =-\sqrt{\frac{a \nu}{1-\alpha t}}(f(\eta)+g(\eta)) \\
\phi & =\frac{C-C_{\infty}}{C_{w}-C_{\infty}}, & \theta & =\frac{T-T_{\infty}}{T_{w}-T_{\infty}}
\end{aligned}
$$

Eq. (1) is satisfied identically and Eqs. (2)-(5) become:

$$
\begin{aligned}
& f^{\prime \prime \prime}-f^{\prime 2}-\zeta\left(\frac{\eta}{2} f^{\prime \prime}+f^{\prime}\right)+(f+g) f^{\prime \prime} \\
& \quad+K\left[-\zeta\left(\frac{\eta}{2} f^{i v}+2 f^{\prime \prime \prime}\right)+(f+g) f^{i v}\right. \\
& \left.\quad+\left(f^{\prime \prime}-g^{\prime \prime}\right) f^{\prime \prime}-2\left(f^{\prime}+g^{\prime}\right) f^{\prime \prime \prime}\right]=0 \\
& g^{\prime \prime \prime}-g^{2}-\zeta\left(\frac{\eta}{2} g^{\prime \prime}+g^{\prime}\right)+(f+g) g^{\prime \prime} \\
& \quad+K\left[-\zeta\left(\frac{\eta}{2} g^{i v}+2 g^{\prime \prime \prime}\right)+(f+g) g^{i v}\right. \\
& \left.\quad-\left(f^{\prime \prime}-g^{\prime \prime}\right) g^{\prime \prime}-2\left(f^{\prime}+g^{\prime}\right) g^{\prime \prime \prime}\right]=0, \\
& \phi^{\prime \prime}+\operatorname{Sc}(f+g) \phi^{\prime}-\operatorname{Sc} \Gamma \phi-\operatorname{Sc} \frac{\zeta}{2} \eta \phi^{\prime}+\operatorname{ScSr} \theta^{\prime \prime}=0, \\
& \left(1+\frac{4}{3 R_{d}}\right) \theta^{\prime \prime}+\operatorname{Pr}(f+g) \theta^{\prime}-\operatorname{Pr} \frac{\zeta}{2} \eta \theta^{\prime}+\operatorname{Pr} H_{g} \theta \\
& +\operatorname{Pr} D_{f} \phi^{\prime \prime}=0,
\end{aligned}
$$

with boundary conditions:

$$
\begin{aligned}
& f(0)=0, \quad g(0)=0, \quad f^{\prime}(0)=1, \quad g^{\prime}(0)=c, \\
& \phi(0)=1, \quad \theta^{\prime}(0)=-\operatorname{Bi}[1-\theta(0)], \quad f^{\prime}(\infty)=0, \\
& g^{\prime}(\infty)=0, \quad \phi(\infty)=0, \quad \theta(\infty)=0,
\end{aligned}
$$

where $\mathrm{Bi}=\frac{h}{k_{T}} \sqrt{\frac{v(1-\alpha t)}{a}}$ is the Biot number, $c=\frac{b}{a}$ is the stretching ratio, $D_{f}=\frac{D k_{T}\left(C_{w}-C_{\infty}\right)}{c_{s} c_{\rho}\left(T_{w}-T_{\infty}\right)(1-\alpha t)}$ is the Dufour number, $\Gamma=\frac{k_{1}}{a}(1-\alpha t)$ is the chemical reaction parameter, $H_{g}=\frac{\stackrel{Q}{Q}(1-\alpha t)}{a \rho c_{\rho}}$ is the heat generation/absorption parameter, $K=\frac{k_{0} a}{v(1-\alpha t)}$ is the dimensionless viscoelastic parameter, $\operatorname{Pr}=\frac{\nu}{\alpha}$ is the Prandtl number, $R_{d}=\frac{k^{*} k_{T}}{4 \sigma_{0} T_{\infty}^{3}}$ is the radiation parameter, $\mathrm{Sc}=$ 
$\frac{\nu}{D}$ is the Schmidt number, $\operatorname{Sr}=\frac{D k_{T}\left(T_{w}-T_{\infty}\right)}{T_{m} v\left(C_{w}-C_{\infty}\right)}$ is the Soret number, and $\zeta=\frac{\alpha}{a}$ is the unsteady parameter.

Note that the two-dimensional $(g=0)$ case has been recovered when $c=0$. For $c=1$, we can find an axisymmetric case, i.e. $(f=g)$.

The local Sherwood and local Nusselt numbers are defined as follows:

$$
\mathrm{Sh}=\frac{x j_{w}}{D\left(C_{w}-C_{\infty}\right)}, \quad \mathrm{Nu}=\frac{x q_{w}}{k_{T}\left(T_{w}-T_{\infty}\right)},
$$

with:

$$
\begin{aligned}
& j_{w}=-D\left(\frac{\partial C}{\partial z}\right)_{z=0}, \\
& q_{w}=-k_{T}\left(\frac{\partial T}{\partial z}\right)_{z=0}-\frac{4 \sigma_{0}}{3 k^{*}}\left(\frac{\partial T^{4}}{\partial z}\right)_{z=0},
\end{aligned}
$$

where $j_{w}$ and $q_{w}$ are the mass and heat fluxes, respectively. Then, the dimensionless forms of Sherwood and Nusselt numbers are:

$$
\begin{aligned}
& \mathrm{Sh} / \sqrt{\operatorname{Re}}=-\phi^{\prime}(0), \\
& \mathrm{Nu} / \sqrt{\operatorname{Re}}=-\left(1+\frac{4}{3 R_{d}}\right) \theta^{\prime}(0),
\end{aligned}
$$

where $\operatorname{Re}=u_{w} x / \nu$ is the local Reynolds number.

\section{Solution by homotopy analysis method}

In order to obtain the HAM solution, the velocity distributions, $f(\eta)$ and $g(\eta)$, the concentration field $\phi(\eta)$, and the temperature field, $\theta(\eta)$, are expressed by the set of base functions, $\left\{\eta^{k} \exp (-m \eta) / k \geq 0, m \geq 0\right\}$, (see $[28,29])$ :

$$
\begin{aligned}
& f(\eta)=a_{0,0}^{0}+\sum_{m=0}^{\infty} \sum_{k=0}^{\infty} a_{n, m}^{k} \eta^{k} \exp (-m \eta), \\
& g(\eta)=A_{0,0}^{0}+\sum_{m=0}^{\infty} \sum_{k=0}^{\infty} A_{n, m}^{k} \eta^{k} \exp (-m \eta), \\
& \phi(\eta)=\sum_{m=0}^{\infty} \sum_{k=0}^{\infty} b_{n, m}^{k} \eta^{k} \exp (-m \eta), \\
& \theta(\eta)=\sum_{m=0}^{\infty} \sum_{k=0}^{\infty} c_{n, m}^{k} \eta^{k} \exp (-m \eta),
\end{aligned}
$$

where $a_{n, m}^{k}, A_{n, m}^{k}, b_{n, m}^{k}$ and $c_{n, m}^{k}$ are the coefficients and the initial guesses $f_{0}(\eta), g_{0}(\eta), \phi_{0}(\eta)$ and $\theta_{0}(\eta)$ are (see [3]):

$$
\begin{aligned}
& f_{0}(\eta)=1-e^{-\eta}, \\
& g_{0}(\eta)=c\left(1-e^{-\eta}\right),
\end{aligned}
$$

$$
\begin{aligned}
& \phi_{0}(\eta)=e^{-\eta}, \\
& \theta_{0}(\eta)=\frac{\mathrm{Bi} e^{-\eta}}{1+\mathrm{Bi}} .
\end{aligned}
$$

The auxiliary linear operators are given by:

$$
\begin{aligned}
& L_{f}=\frac{d^{3} f}{d \eta^{3}}-\frac{d f}{d \eta}, \\
& L_{g}=\frac{d^{3} g}{d \eta^{3}}-\frac{d g}{d \eta} \\
& L_{\phi}=\frac{d^{2} \phi}{d \eta^{2}}-\phi, \\
& L_{\theta}=\frac{d^{2} \theta}{d \eta^{2}}-\theta,
\end{aligned}
$$

with the properties:

$$
\begin{aligned}
& L_{f}\left[C_{1}+C_{2} e^{\eta}+C_{3} e^{-\eta}\right]=0, \\
& L_{g}\left[C_{4}+C_{5} e^{\eta}+C_{6} e^{-\eta}\right]=0, \\
& L_{\phi}\left[C_{7} e^{\eta}+C_{8} e^{-\eta}\right]=0, \\
& L_{\theta}\left[C_{9} e^{\eta}+C_{10} e^{-\eta}\right]=0,
\end{aligned}
$$

in which $C_{i}(i=1-10)$ denote the arbitrary constants.

\subsection{Zero-order deformation problem}

These problems at the zeroth order are:

$$
\begin{aligned}
& (1-p) L_{f}\left[\bar{f}(\eta, p)-f_{0}(\eta)\right]=p h_{f} N_{1}[\bar{f}(\eta, p), \bar{g}(\eta, p)] \\
& (1-p) L_{g}\left[\bar{g}(\eta, p)-g_{0}(\eta)\right]=p h_{g} N_{2}[\bar{f}(\eta, p), \bar{g}(\eta, p)], \\
& (1-p) L_{\phi}\left[\bar{\phi}(\eta, p)-\phi_{0}(\eta)\right] \\
& =p h_{\phi} N_{3}[\bar{f}(\eta, p), \bar{g}(\eta, p), \bar{\phi}(\eta, p), \bar{\theta}(\eta, p)] \\
& (1-p) L_{\theta}\left[\bar{\theta}(\eta, p)-\theta_{0}(\eta)\right] \\
& =p h_{\theta} N_{4}[\bar{f}(\eta, p), \bar{g}(\eta, p), \bar{\phi}(\eta, p), \bar{\theta}(\eta, p)]
\end{aligned}
$$

where $p \in[0,1]$ is an embedding parameter and $h_{f}, h_{g}$, $h_{\phi}$, and $h_{\theta}$ are non-zero auxiliary parameters.

$$
\begin{array}{lll}
\bar{f}(0, p)=0, & \overline{f^{\prime}}(0, p)=1, & \bar{f}(\infty, p)=0, \\
\bar{g}(0, p)=0, & \overline{g^{\prime}}(0, p)=c, & \bar{g}(\infty, p)=0, \\
\bar{\phi}(0, p)=1, \quad \bar{\phi}(\infty, p)=0, & \\
\bar{\theta}(0, p)=-\operatorname{Bi}[1-\theta(0, p)], & \bar{\theta}(\infty, p)=0 .
\end{array}
$$


Further, we define the non-linear operators as follows:

$$
\begin{aligned}
N_{1}[\bar{f}(\eta, p), \bar{g}(\eta, p)]=\frac{\partial^{3} \bar{f}(\eta, p)}{\partial \eta^{3}}-\left(\frac{\partial \bar{f}(\eta, p)}{\partial \eta}\right)^{2} \\
+\zeta\left(\frac{\eta}{2} \frac{\partial^{2} \bar{f}(\eta, p)}{\partial \eta^{2}}+\frac{\partial \bar{f}(\eta, p)}{\partial \eta}\right) \\
+(\bar{f}(\eta, p)+\bar{g}(\eta, p)) \frac{\partial^{2} \bar{f}(\eta, p)}{\partial \eta^{2}} \\
+K \zeta\left(\frac{\eta}{2} \frac{\partial^{4} \bar{f}(\eta, p)}{\partial \eta^{4}}+2 \frac{\partial^{3} \bar{f}(\eta, p)}{\partial \eta^{3}}\right) \\
+K\left[(\bar{f}(\eta, p)+\bar{g}(\eta, p)) \frac{\partial^{4} \bar{f}(\eta, p)}{\partial \eta^{4}}\right. \\
+\left(\frac{\partial^{2} \bar{f}(\eta, p)}{\partial \eta^{2}}-\frac{\partial^{2} \bar{g}(\eta, p)}{\partial \eta^{2}}\right) \frac{\partial^{2} \bar{f}(\eta, p)}{\partial \eta^{2}} \\
\left.+2\left(\frac{\partial \bar{f}(\eta, p)}{\partial \eta}+\frac{\partial \bar{g}(\eta, p)}{\partial \eta}\right) \frac{\partial^{3} \bar{f}(\eta, p)}{\partial \eta^{3}}\right]
\end{aligned}
$$

$$
\begin{aligned}
N_{2} & {[f(\eta, p), \bar{g}(\eta, p)]=\frac{\partial^{3} \bar{g}(\eta, p)}{\partial \eta^{3}}-\left(\frac{\partial \bar{g}(\eta, p)}{\partial \eta}\right)^{2} } \\
& -\zeta\left(\frac{\eta}{2} \frac{\partial^{2} \bar{g}(\eta, p)}{\partial \eta^{2}}+\frac{\partial \bar{g}(\eta, p)}{\partial \eta}\right) \\
+ & (\bar{f}(\eta, p)+\bar{g}(\eta, p)) \frac{\partial^{2} \bar{g}(\eta, p)}{\partial \eta^{2}} \\
& -K \zeta\left(\frac{\eta}{2} \frac{\partial^{4} \bar{g}(\eta, p)}{\partial \eta^{4}}+2 \frac{\partial^{3} \bar{g}(\eta, p)}{\partial \eta^{3}}\right) \\
+ & K\left[(\bar{f}(\eta, p)+\bar{g}(\eta, p)) \frac{\partial^{4} \bar{g}(\eta, p)}{\partial \eta^{4}}\right. \\
& -\left(\frac{\partial^{2} \bar{f}(\eta, p)}{\partial \eta^{2}}-\frac{\partial^{2} \bar{g}(\eta, p)}{\partial \eta^{2}}\right) \frac{\partial^{2} \bar{g}(\eta, p)}{\partial \eta^{2}} \\
& \left.-2\left(\frac{\partial \bar{f}(\eta, p)}{\partial \eta}+\frac{\partial \bar{g}(\eta, p)}{\partial \eta}\right) \frac{\partial^{3} \bar{g}(\eta, p)}{\partial \eta^{3}}\right]
\end{aligned}
$$

$$
\begin{aligned}
N_{3} & {[\bar{f}(\eta, p), \bar{g}(\eta, p), \bar{\phi}(\eta, p), \bar{\theta}(\eta, p)]=\frac{\partial^{2} \bar{\phi}(\eta, p)}{\partial \eta^{2}} } \\
+ & \operatorname{Sc}(\bar{f}(\eta, p)+\bar{g}(\eta, p)) \frac{\partial \bar{\phi}(\eta, p)}{\partial \eta}-\operatorname{Sc} \Gamma \bar{\phi}(\eta, p) \\
& -\operatorname{Sc} \frac{\zeta}{2} \eta \frac{\partial \bar{\phi}(\eta, p)}{\partial \eta}+\operatorname{ScSr} \frac{\partial^{2} \bar{\theta}(\eta, p)}{\partial \eta^{2}}
\end{aligned}
$$

$N_{4}[\bar{f}(\eta, p), \bar{g}(\eta, p), \bar{\phi}(\eta, p), \bar{\theta}(\eta, p)]$

$$
\begin{aligned}
& =\left(1+\frac{4}{3 R_{d}}\right) \frac{\partial^{2} \bar{\theta}(\eta, p)}{\partial \eta^{2}}-\operatorname{Pr} \frac{\zeta}{2} \eta \frac{\partial \bar{\theta}(\eta, p)}{\partial \eta} \\
& +\operatorname{Pr}(\bar{f}(\eta, p)+\bar{g}(\eta, p)) \frac{\partial \bar{\theta}(\eta, p)}{\partial \eta}+\operatorname{Pr} H_{g} \bar{\theta}(\eta, p) \\
& +\operatorname{Pr} D_{f} \frac{\partial^{2} \bar{\phi}(\eta, p)}{\partial \eta^{2}}
\end{aligned}
$$

For $p=0$ and $p=1$, we can define:

$$
\begin{array}{ll}
\bar{f}(\eta, 0)=f_{0}(\eta), & \bar{f}(\eta, 1)=f(\eta), \\
\bar{g}(\eta, 0)=g_{0}(\eta), & \bar{g}(\eta, 1)=g(\eta), \\
\bar{\phi}(\eta, 0)=\phi_{0}(\eta), & \bar{\phi}(\eta, 1)=\phi(\eta), \\
\bar{\theta}(\eta, 0)=\theta_{0}(\eta), & \bar{\theta}(\eta, 1)=\theta(\eta) .
\end{array}
$$

The initial guesses $f_{0}(\eta), g_{0}(\eta), \phi_{0}(\eta)$, and $\theta_{0}(\eta)$ approach $f(\eta), g(\eta), \phi(\eta)$, and $\theta(\eta)$, respectively, when $p$ varies from 0 to 1 .

By Taylor's series expansion, we have:

$$
\begin{aligned}
& \bar{f}(\eta, p)=f_{0}(\eta)+\sum_{m=1}^{\infty} f_{m}(\eta) p^{m} \\
& \bar{g}(\eta, p)=g_{0}(\eta)+\sum_{m=1}^{\infty} g_{m}(\eta) p^{m} \\
& \bar{\phi}(\eta, p)=\phi_{0}(\eta)+\sum_{m=1}^{\infty} \phi_{m}(\eta) p^{m} \\
& \bar{\theta}(\eta, p)=\theta_{0}(\eta)+\sum_{m=1}^{\infty} \theta_{m}(\eta) p^{m}
\end{aligned}
$$

where:

$$
\begin{aligned}
& f_{m}(\eta)=\left.\frac{1}{m !} \frac{\partial^{m} \bar{f}(\eta, p)}{\partial p^{m}}\right|_{p=0}, \\
& g_{m}(\eta)=\left.\frac{1}{m !} \frac{\partial^{m} \bar{g}(\eta, p)}{\partial p^{m}}\right|_{p=0}, \\
& \phi_{m}(\eta)=\left.\frac{1}{m !} \frac{\partial^{m} \bar{\phi}(\eta, p)}{\partial p^{m}}\right|_{p=0}, \\
& \theta_{m}(\eta)=\left.\frac{1}{m !} \frac{\partial^{m} \bar{\theta}(\eta, p)}{\partial p^{m}}\right|_{p=0} .
\end{aligned}
$$

The series in the above four equations strongly depends on $h_{f}, h_{g}, h_{\phi}$, and $h_{\theta}$. The values of the auxiliary 
parameters $h_{f}, h_{g}, h_{\phi}$, and $h_{\theta}$ are chosen by a process to converge at $p=1$. Hence:

$$
\begin{aligned}
& f(\eta)=f_{0}(\eta)+\sum_{m=1}^{\infty} f_{m}(\eta), \\
& g(\eta)=g_{0}(\eta)+\sum_{m=1}^{\infty} g_{m}(\eta), \\
& \phi(\eta)=\phi_{0}(\eta)+\sum_{m=1}^{\infty} \phi_{m}(\eta), \\
& \theta(\eta)=\theta_{0}(\eta)+\sum_{m=1}^{\infty} \theta_{m}(\eta) .
\end{aligned}
$$

\subsection{Higher-order deformation problem}

The $m$ th-order deformation problem is of the form:

$$
\begin{aligned}
& L_{f}\left[f_{m}(\eta)-\chi_{m} f_{m-1}(\eta)\right]=h_{f} R_{1, m}(\eta), \\
& L_{g}\left[g_{m}(\eta)-\chi_{m} g_{m-1}(\eta)\right]=h_{g} R_{2, m}(\eta), \\
& L_{\phi}\left[\phi_{m}(\eta)-\chi_{m} \phi_{m-1}(\eta)\right]=h_{\phi} R_{3, m}(\eta), \\
& L_{\theta}\left[\theta_{m}(\eta)-\chi_{m} \theta_{m-1}(\eta)\right]=h_{\theta} R_{4, m}(\eta), \\
& f_{m}(0)=f_{m}^{\prime}(0)=f_{m}^{\prime}(\infty)=g_{m}^{\prime}(\infty)=g_{m}(0)=g_{m}^{\prime}(0)=0, \\
& \theta_{m}^{\prime}(0)-\operatorname{Bi} \theta_{m}(0)=0, \\
& \phi_{m}(0)=\phi_{m}(\infty)=\theta_{m}(\infty)=0 .
\end{aligned}
$$

The values of $R_{i, m}(i=1,2,3,4)$ are defined as follows:

$$
\begin{aligned}
R_{1, m}= & f_{m-1}^{\prime \prime \prime}-\zeta\left(\frac{\eta}{2} f_{m-1}^{\prime \prime}+f_{m-1}^{\prime}\right) \\
& -K \zeta\left(\frac{\eta}{2} f_{m-1}^{i v}+2 f_{m-1}^{\prime \prime \prime}\right) \\
& +\sum_{k=0}^{m-1}\left\{\left(f_{m-1-k}+g_{m-k-1}\right) f_{k}^{\prime \prime}\right. \\
& -f_{m-1-k}^{\prime} f_{k}^{\prime}+K\left[\left(f_{m-1-k}+g_{m-k-1}\right) f_{k}^{i v}\right. \\
& +\left(f_{m-1-k}^{\prime \prime}-g_{m-k-1}^{\prime \prime}\right) f_{k}^{\prime \prime} \\
& \left.\left.-2\left(f_{m-1-k}^{\prime}+g_{m-k-1}^{\prime}\right) f_{k}^{\prime \prime \prime}\right]\right\}, \\
R_{2, m} & g_{m-1}^{\prime \prime \prime}-\zeta\left(\frac{\eta}{2} g_{m-1}^{\prime \prime}+g_{m-1}^{\prime}\right) \\
& -K \zeta\left(\frac{\eta}{2} g_{m-1}^{i v}+2 g_{m-1}^{\prime \prime \prime}\right) \\
& +\sum_{k=0}^{m-1}\left\{\left(f_{m-1-k}+g_{m-k-1}\right) g_{k}^{\prime \prime}-g_{m-1-k}^{\prime} g_{k}^{\prime}\right. \\
& +K\left[\left(f_{m-1-k}+g_{m-k-1}\right) g_{k}^{i v}\right. \\
& -61) \\
&
\end{aligned}
$$

$$
\begin{aligned}
&-\left(f_{m-k-1}^{\prime \prime}-g_{m-1-k}^{\prime \prime}\right) g_{k}^{\prime \prime} \\
&\left.\left.-2\left(f_{m-1-k}^{\prime}+g_{m-k-1}^{\prime}\right) g_{k}^{\prime \prime \prime}\right]\right\}, \\
& R_{3, m}= \phi_{m-1}^{\prime \prime}+\operatorname{Sc} \sum_{k=0}^{m-1}\left(f_{m-1-k}+g_{m-k-1}\right) \phi_{k}^{\prime} \\
&-\operatorname{Sc} \Gamma \phi_{m-1}-\operatorname{Sc} \frac{\zeta}{2} \eta \phi_{m-1}^{\prime}+\operatorname{ScSr} \theta_{m-1}^{\prime \prime}, \\
& R_{4, m}=\left(1+\frac{4}{3 R_{d}}\right) \theta_{m-1}^{\prime \prime} \\
&+\operatorname{Pr} \sum_{k=0}^{m-1}\left(f_{m-1-k}+g_{m-k-1}\right) \theta_{k}^{\prime} \\
&-\operatorname{Pr} \frac{\zeta}{2} \eta \theta_{m-1}^{\prime}+\operatorname{Pr} H_{g} \theta_{m-1}+\operatorname{Pr} D_{f} \phi_{m-1}^{\prime \prime}, \\
& \chi_{m}= \begin{cases}0, & m \leq 1 \\
1, & m>1\end{cases}
\end{aligned}
$$

Finally, the general solutions to Eqs. (56)-(59) can be written as:

$$
\begin{aligned}
& f_{m}(\eta)=f_{m}^{*}(\eta)+C_{1}+C_{2} e^{\eta}+C_{3} e^{-\eta}, \\
& g_{m}(\eta)=g_{m}^{*}(\eta)+C_{4}+C_{5} e^{\eta}+C_{6} e^{-\eta}, \\
& \phi_{m}(\eta)=\phi_{m}^{*}(\eta)+C_{7} e^{\eta}+C_{8} e^{-\eta}, \\
& \theta_{m}(\eta)=\theta_{m}^{*}(\eta)+C_{9} e^{\eta}+C_{10} e^{-\eta},
\end{aligned}
$$

where $f_{m}^{*}(\eta), g_{m}^{*}(\eta), \phi_{m}^{*}(\eta)$, and $\theta_{m}^{*}(\eta)$ are the particular solutions to Eqs. (56)-(59) and the coeffiecients $C_{i}(i=1,2,3, \cdots, 10)$ are determined by the boundary conditions $(60)$.

The symbolic computations are carried out by MATLAB.

\subsection{Convergence of the homotopy analysis method}

Obviously, the series solution contains the non-zero auxiliary parameters $h_{f}, h_{g}, h_{\phi}$, and $h_{\theta}$. Such parameters adjust and control the convergence of the HAM solutions. In order to find the range for admissible values of $h_{f}, h_{g}, h_{\phi}$, and $h_{\theta}$, the $h$ curves of the functions $f^{\prime \prime}(0), g^{\prime \prime}(0), \phi^{\prime}(0)$, and $\theta^{\prime}(0)$ are plotted. Figure 1(a) indicates that the respective admissible values of $h_{f}$ and $h_{g}$ are $-1.2 \leq h_{f} \leq-0.4$ and $-1.3 \leq h_{g} \leq-0.4$. Figure $1(\mathrm{~b})$ shows that the possible values of $h_{\phi}$ and $h_{\theta}$ are $-1.5 \leq h_{\phi} \leq-0.4$ and $-1.5 \leq h_{\theta} \leq-0.4$. If we choose the values of auxiliary parameter $h$ from this range, we will get more accurate results. Further, the series also converge in the whole region of $\eta$ when $h_{f}=h_{g}=h_{\phi}=h_{\theta}=-0.9$.

Using this $h$ value, we get the solution of the present problem for different parameters. 


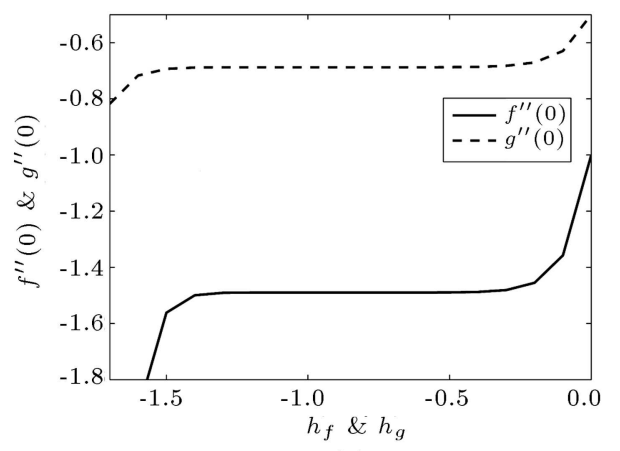

(a)

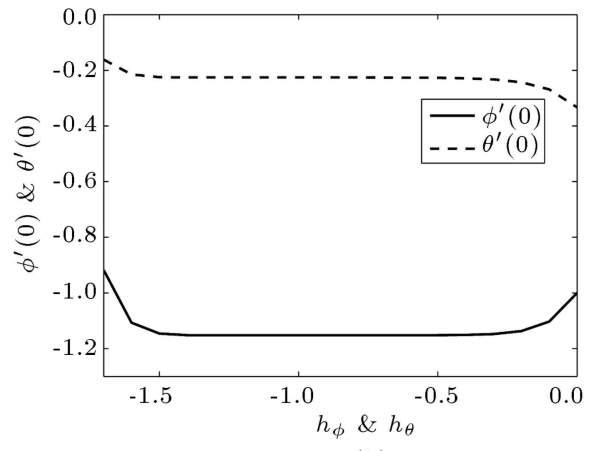

(b)

Figure 1. $h$ curves of $f^{\prime \prime} \& g^{\prime \prime}(\mathrm{a})$ and $\phi^{\prime} \& \theta^{\prime}(\mathrm{b})$ with $\zeta=0.5, K=0.1, c=0.5, \mathrm{Sr}=0.3, \Gamma=1.0, H_{g}=-0.5, D_{f}=0.3$, $\mathrm{Bi}=0.5$, and $R_{d}=10.0$.

Table 1. Comparison of $-f^{\prime \prime}(0)$ and $-g^{\prime \prime}(0)$ for different values of $c$ with those in Hayat et al. [19].

\begin{tabular}{cccccc}
\hline & \multicolumn{2}{c}{$-\boldsymbol{f}^{\prime \prime}(\mathbf{0})$} & & \multicolumn{2}{c}{$-\boldsymbol{g}^{\prime \prime}(\mathbf{0})$} \\
\cline { 2 - 3 } \cline { 6 - 7 } $\boldsymbol{c}$ & $\begin{array}{c}\text { Present } \\
\text { study }\end{array}$ & $\begin{array}{c}\text { Hayat } \\
\text { et al. } \\
{[\mathbf{1 9}]}\end{array}$ & & $\begin{array}{c}\text { Present } \\
\text { study }\end{array}$ & $\begin{array}{c}\text { Hayat } \\
\text { et al. } \\
{[\mathbf{1 9}]}\end{array}$ \\
\hline 0.0 & 1.000000 & 1.000000 & & 0.000000 & 0.000000 \\
0.2 & 1.039495 & 1.039495 & & 0.148737 & 0.148736 \\
0.5 & 1.093095 & 1.093035 & & 0.465205 & 0.465204 \\
0.7 & 1.126398 & 1.126397 & & 0.724532 & 0.724531 \\
1.0 & 1.173712 & 1.173720 & & 1.173712 & 1.173720 \\
\hline
\end{tabular}

\section{Results and discussion}

In this section, we show the influence of different parameters on velocity profiles $\left(f^{\prime} \& g^{\prime}\right)$, concentration profile $(\phi)$, and temperature profile $(\theta)$ for the fixed values of the Prandtl number $(\operatorname{Pr}=0.7)$ and Schmidt number $(\mathrm{Sc}=1.0)$. The parameters involved in the study are the Biot number $(\mathrm{Bi})$, viscoelastic parameter $(K)$, stretching ratio $(c)$, unsteadiness parameter $(\zeta)$, Soret number $(\mathrm{Sr})$, chemical reaction parameter $(\Gamma)$, radiation parameter $\left(R_{d}\right)$, Dufour number $\left(D_{f}\right)$, and heat generation/absorption parameter $\left(H_{g}\right)$.

Table 1 provides the comparison of the results for different values of $c$ with the results presented by Hayat et al. [19]. It is observed from the table that our results agree well with Hayat et al. [19]. This shows the certainty of our solution. Table 2 shows the values of $-f^{\prime \prime}(0),-g^{\prime \prime}(0),-\phi^{\prime}(0)$, and $-\theta^{\prime}(0)$ for different values of $\zeta, c$, and $K$. Increasing the values of $\zeta$ and $K$ increases $-f^{\prime \prime}$ and $-g^{\prime \prime}$ and decreases $-\phi^{\prime}$ and $-\theta^{\prime}$, respectively. It is also found that $-f^{\prime \prime}$ and $-g^{\prime \prime}$, and $-\phi^{\prime}$ and $-\theta^{\prime}$ are increasing functions of $c$. Table 3 provides the values of local Sherwood number and local Nusselt number for various combinations of physical parameters involved in the study. It is noted that the local Sherwood number increases on increasing $D_{f}, H_{g}$, and $\Gamma$ values and it decreases with increasing the values of $\mathrm{Sr}, R_{d}$, and Bi. In addition, the local Nusselt number
Table 2. Variations of $-f^{\prime \prime}(0),-g^{\prime \prime}(0),-\phi^{\prime}(0)$ and $-\theta^{\prime}(0)$ for different parameters with $\mathrm{Sr}=0.3, D_{f}=0.3, \mathrm{Pr}=0.7$, $\mathrm{Sc}=1.0, H_{g}=-0.5, R_{d}=10, \mathrm{Bi}=0.5$, and $\Gamma=1.0$.

\begin{tabular}{ccccccc}
\hline $\boldsymbol{\zeta}$ & $\boldsymbol{c}$ & $\boldsymbol{K}$ & $-\boldsymbol{f}^{\prime \prime}(\mathbf{0})$ & $-\boldsymbol{g}^{\prime \prime}(\mathbf{0})$ & $-\boldsymbol{\phi}^{\prime}(\mathbf{0})$ & $-\boldsymbol{\theta}^{\prime}(\mathbf{0})$ \\
\hline 0.0 & 0.5 & 0.1 & 1.221549 & 0.538076 & 1.232802 & 0.243544 \\
0.3 & & & 1.378843 & 0.625724 & 1.184895 & 0.233374 \\
0.6 & & & 1.547220 & 0.719642 & 1.135729 & 0.221996 \\
0.9 & & & 1.727996 & 0.820336 & 1.086554 & 0.210652 \\
0.1 & 0.0 & 0.1 & 1.102878 & 0.000000 & 1.143444 & 0.231324 \\
& 0.5 & & 1.272796 & 0.566604 & 1.217016 & 0.240333 \\
& 1.0 & & 1.465625 & 1.465625 & 1.278367 & 0.246355 \\
& 1.5 & & 1.704913 & 2.665794 & 1.325867 & 0.250646 \\
0.1 & 0.5 & 0.0 & 1.122876 & 0.481927 & 1.227980 & 0.242883 \\
& & 0.1 & 1.272796 & 0.566604 & 1.217016 & 0.240333 \\
& & 0.2 & 1.532326 & 0.753884 & 1.200321 & 0.236781 \\
& & 0.3 & 2.364099 & 1.737231 & 1.159027 & 0.229863 \\
\hline
\end{tabular}

increases on increasing $\mathrm{Sr}$ and $\mathrm{Bi}$ values and it decreases on increasing $D_{f}, H_{g}, R_{d}$, and $\Gamma$ values.

The effects of unsteadiness parameter $(\zeta)$ on velocity are displayed in Figure 2(a) and (b). It is observed that the velocity and its boundary layer thickness decrease as the unsteadiness parameter increases. This inhibits the growth of change of laminar flow to turbulent flow. Figure $3(\mathrm{a})$ and (b) display the variations of viscoelastic parameter $(K)$ on velocity. It is seen from these figures that increase in viscoelastic parameter brings a decrease in the fluid velocity. The influence of stretching ratio $(c)$ on velocity is displayed in Figure 4(a) and (b). It is observed that the $x$ component velocity and its boundary layer thickness decrease with an increase in the stretching ratio. However $y$-component velocity and its boundary layer thickness increase on increasing the stretching ratio.

Figure 5 depicts the effects of Soret number on the concentration distribution. It can be clearly seen from this figure that the thermal diffusion effects slightly affect the fluid concentration. As the value of the Soret number increases, the concentration of 


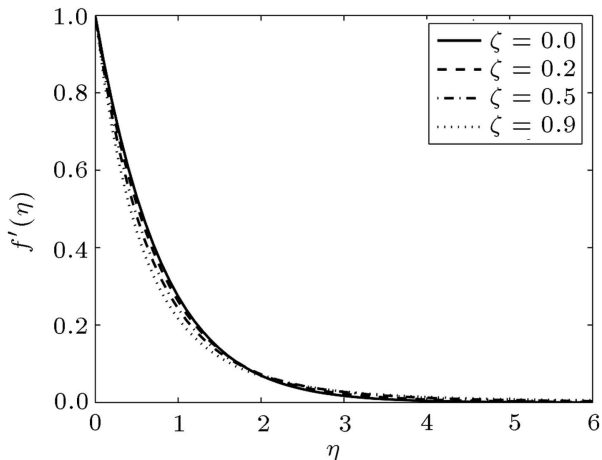

(a)

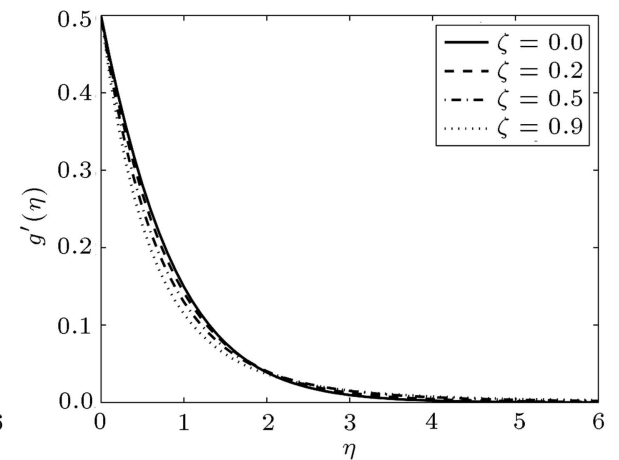

(b)

Figure 2. (a) $x$-component velocity $\left(f^{\prime}\right)$, and (b) $y$-component velocity $\left(g^{\prime}\right)$ for different values of $\zeta$ with $K=0.1, c=0.5$, $\mathrm{Sr}=0.3, \Gamma=1.0, H_{g}=-0.5, D_{f}=0.3, \mathrm{Bi}=0.5$, and $R_{d}=10.0$.

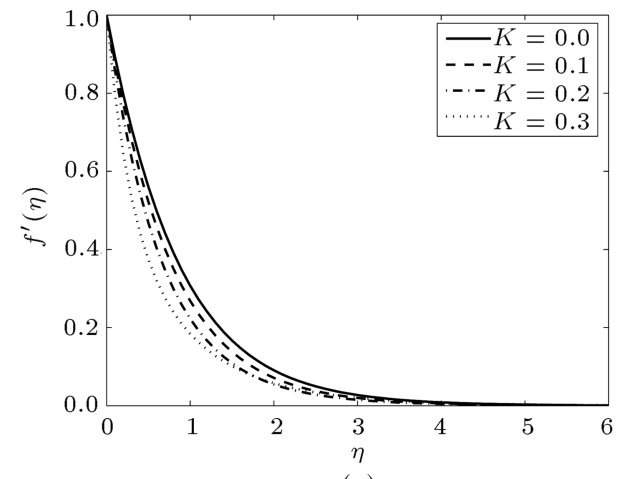

(a)

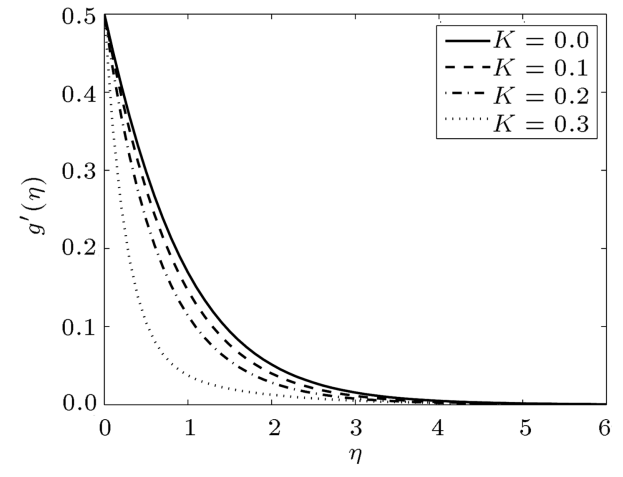

(b)

Figure 3. (a) $x$-component velocity $\left(f^{\prime}\right)$, and (b) $y$-component velocity $\left(g^{\prime}\right)$ for different values of $K$ with $\zeta=0.5, c=0.5$, $\mathrm{Sr}=0.3, \Gamma=1.0, H_{g}=-0.5, D_{f}=0.3, \mathrm{Bi}=0.5$, and $R_{d}=10.0$.

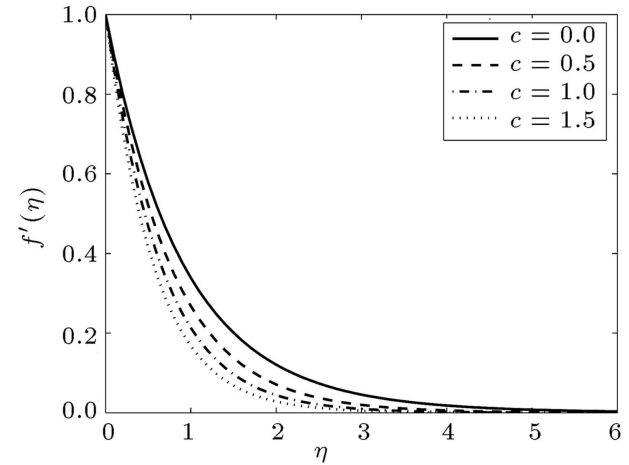

(a)

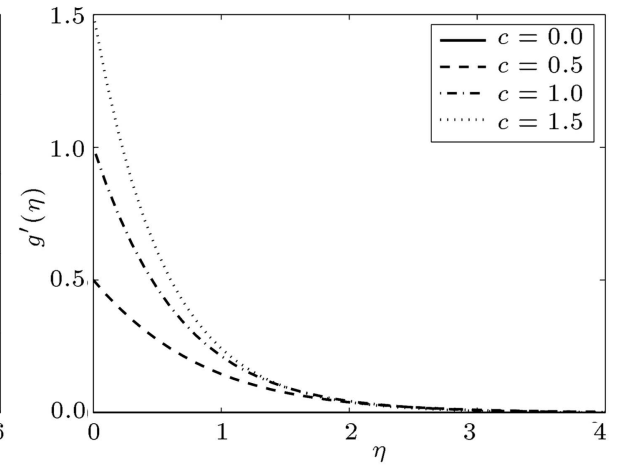

(b)

Figure 4. (a) $x$-component velocity $\left(f^{\prime}\right)$, and (b) $y$-component velocity $\left(g^{\prime}\right)$ for different values of $c$ with $\zeta=0.5, K=0.1$, $\mathrm{Sr}=0.3, \Gamma=1.0, H_{g}=-0.5, D_{f}=0.3, \mathrm{Bi}=0.5$, and $R_{d}=10.0$.

the fluid and solutal boundary layer thickness increase. Figure 6 shows the effect of the chemical reaction $(\Gamma)$ on concentration profile. Increasing the chemical reaction produces a decrease in the species concentration. The influence of heat generation/absorption parameter $\left(H_{g}\right)$ on temperature is shown in Figure 7 . The effect of internal heat generation $\left(H_{g}>0\right)$ is to increase the rate of heat transport to the fluid, thereby increasing the temperature of the fluid. However, heat absorption $\left(H_{g}<0\right)$ is to decrease the rate of heat transport to the fluid, thereby decreasing the temperature of the fluid. The temperature profile for different values of Dufour number $\left(D_{f}\right)$ is shown in Figure 8. It is noticed that the diffusion thermal effects greatly affect the fluid temperature. An increase in Dufour number corresponds to an increase in the fluid temperature and thermal boundary layer thickness. The variation of Biot number ( $\mathrm{Bi}$ ) on temperature is illustrated in Figure 9. It is observed that the Biot number yields an increase in the fluid temperature and 
Table 3. Local Sherwood and Nusselt numbers for different parameters with $\zeta=0.1, c=0.5, K=0.1$, $\operatorname{Pr}=0.7$ and $\mathrm{Sc}=1.0$.

\begin{tabular}{|c|c|c|c|c|c|c|c|}
\hline $\mathrm{Sr}$ & $D_{f}$ & $\boldsymbol{H}_{g}$ & $\boldsymbol{R}_{d}$ & $\Gamma$ & $\mathrm{Bi}$ & $\mathrm{Sh} / \sqrt{\mathrm{Re}}$ & $\mathrm{Nu} / \sqrt{\mathbf{R e}}$ \\
\hline 0.0 & 0.3 & -0.5 & 10 & 1.0 & 0.5 & 1.233690 & 0.271441 \\
\hline 0.3 & & & & & & 1.217016 & 0.272377 \\
\hline 0.6 & & & & & & 1.199925 & 0.273341 \\
\hline 0.9 & & & & & & 1.182402 & 0.274332 \\
\hline \multirow[t]{4}{*}{0.3} & 0.0 & -0.5 & 10 & 1.0 & 0.5 & 1.195806 & 0.341285 \\
\hline & 0.3 & & & & & 1.217016 & 0.272377 \\
\hline & 0.6 & & & & & 1.239736 & 0.199913 \\
\hline & 0.9 & & & & & 1.264170 & 0.123461 \\
\hline \multirow[t]{5}{*}{0.3} & 0.3 & -1.5 & 10 & 1.0 & & 1.193898 & 0.348145 \\
\hline & & -1.0 & & & & 1.203150 & 0.319421 \\
\hline & & -0.5 & & & & 1.217016 & 0.272378 \\
\hline & & 0.0 & & & & 1.244350 & 0.161277 \\
\hline & & 0.5 & & & & 1.356229 & -0.545321 \\
\hline \multirow[t]{5}{*}{0.3} & 0.3 & -0.5 & 1 & 1.0 & 0.5 & 1.217995 & 0.472876 \\
\hline & & & 3 & & & 1.217866 & 0.332504 \\
\hline & & & 5 & & & 1.217672 & 0.298666 \\
\hline & & & 8 & & & 1.217185 & 0.279028 \\
\hline & & & 10 & & & 1.217016 & 0.272377 \\
\hline \multirow[t]{5}{*}{0.3} & 0.3 & -0.5 & 10 & -0.5 & 0.5 & -1.098295 & 0.398333 \\
\hline & & & & 0.0 & & 0.602734 & 0.315884 \\
\hline & & & & 0.5 & & 0.966337 & 0.290624 \\
\hline & & & & 1.0 & & 1.217016 & 0.272377 \\
\hline & & & & 1.5 & & 1.421756 & 0.257145 \\
\hline \multirow[t]{5}{*}{0.3} & 0.3 & -0.5 & 10 & 1.0 & 0.1 & 1.238788 & 0.079460 \\
\hline & & & & & 0.3 & 1.225871 & 0.193912 \\
\hline & & & & & 0.5 & 1.217016 & 0.272377 \\
\hline & & & & & 0.8 & 1.207958 & 0.352698 \\
\hline & & & & & 1.0 & 1.203623 & 0.391056 \\
\hline
\end{tabular}

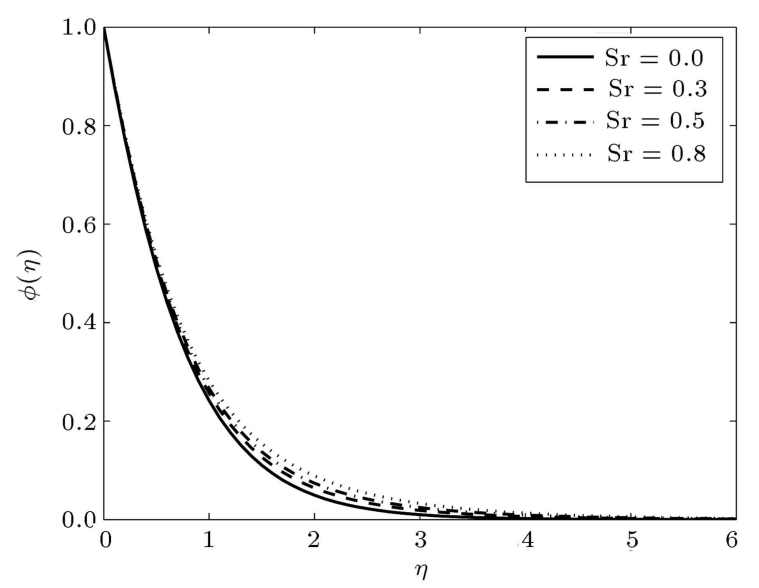

Figure 5. Concentration $(\phi)$ for different values of $\mathrm{Sr}$ with $\zeta=0.5, K=0.1, c=0.5, \Gamma=1.0, H_{g}=-0.5$, $D_{f}=0.3, \mathrm{Bi}=0.5$, and $R_{d}=10.0$.

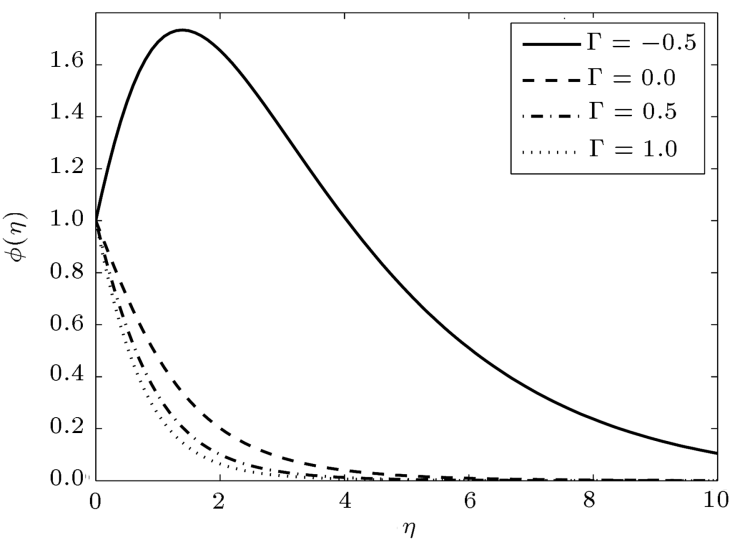

Figure 6. Concentration $(\phi)$ for different values of $\Gamma$ with $\zeta=0.5, K=0.1, c=0.5, \mathrm{Sr}=0.3, H_{g}=-0.5, D_{f}=0.3$, $\mathrm{Bi}=0.5$, and $R_{d}=10.0$.

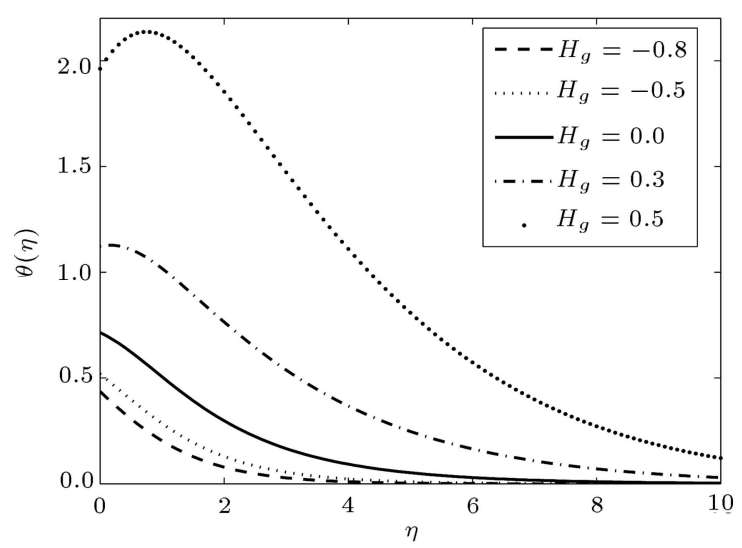

Figure 7. Temperature $(\theta)$ for different values of $H_{g}$ with $\zeta=0.5, K=0.1, c=0.5, \mathrm{Sr}=0.3, \Gamma=1.0, D_{f}=0.3$, $\mathrm{Bi}=0.5$, and $R_{d}=10.0$.

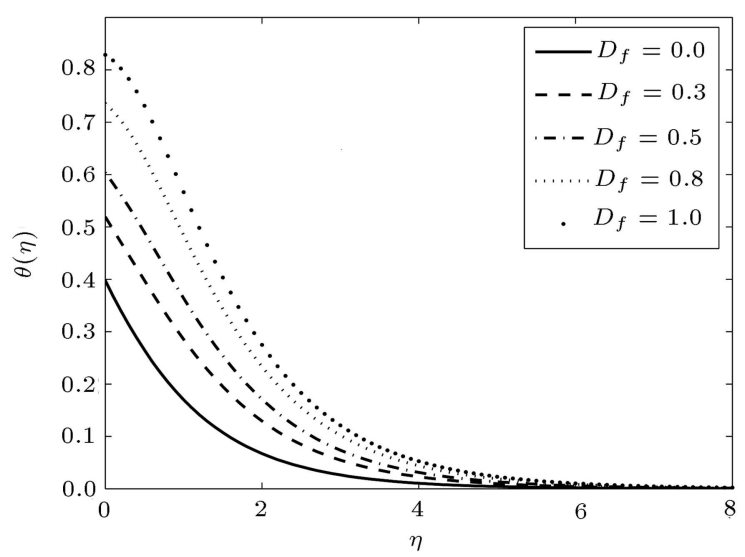

Figure 8. Temperature $(\theta)$ for different values of $D_{f}$ with $\zeta=0.5, K=0.1, c=0.5, \mathrm{Sr}=0.3, \Gamma=1.0, H_{g}=-0.5$, $\mathrm{Bi}=0.5$, and $R_{d}=10.0$.

thermal boundary layer thickness. Figure 10 presents the effect of radiation parameter $\left(R_{d}\right)$ on temperature. It is observed that the fluid temperature decreases with an increase in the thermal radiation. These results quantitatively agree with expectations, since the effect 


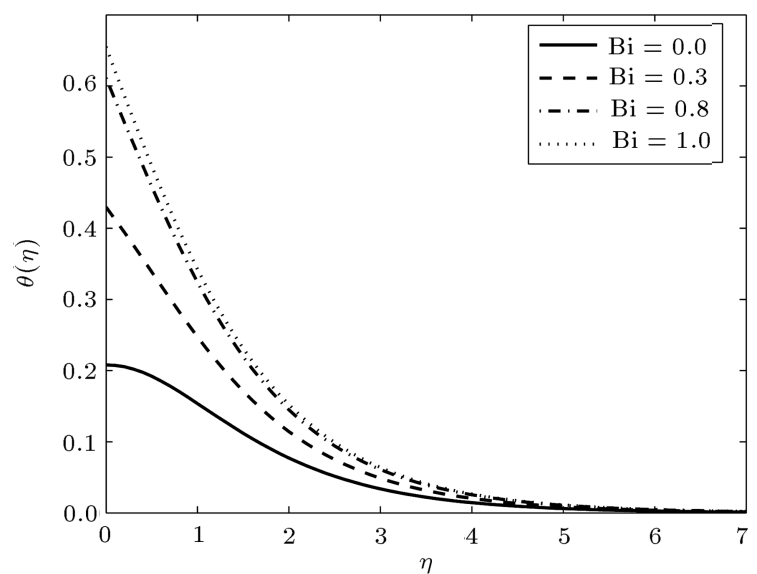

Figure 9. Temperature $(\theta)$ for different values of Bi with $\zeta=0.5, K=0.1, c=0.5, \mathrm{Sr}=0.3, \Gamma=1.0, H_{g}=-0.5$, $D_{f}=0.3$, and $R_{d}=10.0$.

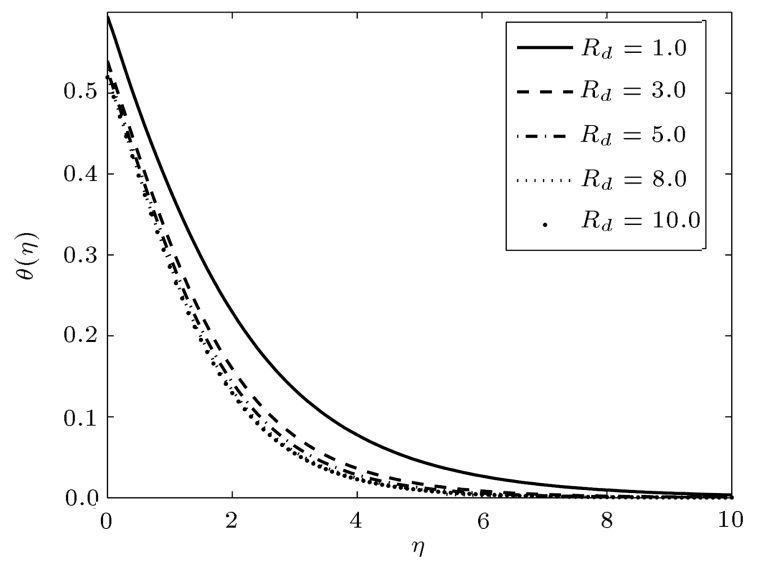

Figure 10. Temperature $(\theta)$ for different values of $R_{d}$ with $\zeta=0.5, K=0.1, c=0.5, \mathrm{Sr}=0.3, \Gamma=1.0$, $H_{g}=-0.5, D_{f}=0.3$, and $\mathrm{Bi}=0.5$.

of radiation decreases the rate of energy transport to the fluid, thereby decreasing the fluid temperature.

\section{Conclusion}

The present study deals with the Soret and Dufour effects on unsteady three-dimensional boundary layer flow, and heat and mass transfer over a stretching surface with convective boundary condition in the presence of thermal radiation and chemical reaction. The governing momentum, concentration, and energy equations are transformed into ordinary differential equations using similarity variables. The equations are solved by the homotopy analysis method and the influences of the parameters on velocity, concentration, and temperature profiles are displayed. The following main observations are obtained:

- The velocity boundary layer thickness decreases with increase in the viscoelastic and unsteady parameters;
- The concentration boundary layer thickness increases on increasing the Soret number;

- The thermal boundary layer thickness increases with increase in the Dufour number and it decreases on increasing the radiation parameter;

- The local Sherwood number increases on increasing the Dufour number, heat generation/absorption, and chemical reaction parameters and it decreases on increasing the Soret number, radiation parameter, and Biot number;

- It is observed that the local Nusselt number increases on increasing the Soret number and Biot number, and it decreases on increasing the Dufour number, heat generation/absorption, radiation, and chemical reaction parameters.

\section{Acknowledgements}

The third author acknowledges University of Malaya for the financial support through the grants RG21612AFR and RP011B-13AFR.

\section{References}

1. Hayat, T., Mustafa, M. and Pop, I. "Heat and mass transfer for Soret and Dufours effects on mixed convection boundary layer flow over a stretching vertical surface in a porous medium filled with a viscoelastic fluid", Commun. Sci. Numer. Simul., 15, pp. 11831196 (2010).

2. Chowdhury, M.K. and Islam, M.N. "MHD free convection flow of viscoelastic fluid past an infinite vertical porous plate", Heat Mass Transf., 36, pp. 439-447 (2000).

3. Hayat, T., Mustafa, M. and Hendi, A.A. "Time dependent three-dimensional flow and mass transfer of elastico-viscous fluid over unsteady stretching sheet", Appl. Math. Mech., (English Ed.) 32(2), pp. 167-178 (2011).

4. Anwar, I., Amin, N. and Pop, I. "Mixed convection boundary layer flow of a viscoelastic fluid over a horizontal circular cylinder", Int. J. Non-Linear Mech., 43, pp. 814-821 (2008).

5. Chin, K.E., Nazar, R., Arifin, N.M. and Pop, I. "Effect of variable viscosity on mixed convection boundary layer flowover a vertical surface embedded in a porous medium", Int. Commun. Heat Mass Transf., 34, pp. 464-473 (2007).

6. Sanjayanand, E. and Khan, S.K. "On heat and mass transfer in a viscoelastic boundary layer flow over an exponentially stretching sheet", Int. J. Therm. Sci., 45, pp. 819-828 (2006).

7. Bhuvaneswari, M., Sivasankaran, S. and Ferdows, M. "Lie group analysis of natural convection heat and mass transfer in an inclined surface with chemical reaction", Nonlinear Anal. Hybrid Syst., 3, pp. 536542 (2009). 
8. Pal, D. and Talukdar, B. "Buoyancy and chemical reaction effects on MHD mixed convection heat and mass transfer in a porous medium with thermal radiation and Ohmic heating", Commun. Sci. Numer. Simul., 15, pp. 2878-2893 (2010).

9. Mozayyeni, H.R. and Rahimi, A.B. "Similarity solutions of axisymmetric stagnation-point flow and heat transfer of a viscous, Boussinesq-related density fluid on a moving at plate", Scientia Iranica B, 21(4), pp. 1440-1450 (2014).

10. Afify, A.A. "MHD free convective flow and mass transfer over a stretching sheet with chemical reaction", Heat Mass Transf., 40, pp. 495-500 (2004).

11. Aboeldahab, E.M. and Azzam, G.E.D.A. "Unsteady three-dimensional combined heat and mass free convective flow over a stretching surface with timedependent chemical reaction", Acta Mech., 184, pp. 121-136 (2006).

12. Hamad, M.A.A., Uddin, Md.J. and Ismai, A.I.Md. "Investigation of combined heat and mass transfer by Lie group analysis with variable diffusivity taking into account hydrodynamic slip and thermal convective boundary conditions", Int. J. Heat Mass Transf., 55, pp. 1355-1362 (2012).

13. Hossain, M.A., Khanafer, K. and Vafai, K. "The effect of radiation on free convection flow of fluid with variable viscosity from a porous vertical plate", Int. J. Therm. Sci., 40, pp. 115-124 (2001).

14. Lee, J., Kandaswamy, P., Bhuvaneswari, M. and Sivasankaran, S. "Lie group analysis of radiation natural convection heat transfer past an inclined porous surface", J. Mech. Sci. Tech., 22, pp. 1779-1784 (2008).

15. Bhuvaneswari, M., Sivasankaran, S. and Kim, Y.J. "Lie group analysis of radiation natural convection flow over an inclined surface in a porous medium with internal heat generation", J. Porous Media, 15(12), pp. 1155-1164 (2012).

16. Bhuvaneswari, M., Sivasankaran, S. and Kim, Y.J. "Exact analysis of radiation convective flow heat and mass transfer over an inclined plate in a porous medium", World Appl. Sci., 10(7), pp. 774-778 (2010).

17. Ahmed, S.E., Hussein, A.K., Mohammed, H.A. and Sivasankaran, S. "Boundary layer flow and heat transfer due to permeable stretching tube in the presence of heat source/sink utilizing nanofluids", Appl. Math. Comput., 238, pp. 149-162 (2014).

18. Postelnicu, A. "Influence of chemical reaction on heat and mass transfer by natural convection from vertical surfaces in porous media considering Soret and Dufour effects", Heat Mass Transf., 43, pp. 595-602 (2007).

19. Hayat, T., Safdar, A., Awas, M. and Mesloub, S. "Soret and Dufour effects for three dimensional flow in a viscoelastic fluid over a stretching surface", Int. J. Heat Mass Transf., 55, pp. 2129-2136 (2012).

20. Chamkha, A.J. and Nakhi, A.B. "MHD mixed convection radiation interaction along a permeable surface immersed in a porous medium in the presence of Soret and Dufour's effects", Heat Mass Transf., 44, pp. 845856 (2008).

21. Postelnicu, A. "Influence of a magnetic field on heat and mass transfer by natural convection from vertical surfaces in porous media considering Soret and Dufour effects", Int. J. Heat Mass Transf., 47, pp. 1467-1472 (2004).

22. Rathish Kumar, B.V.S. and Krishna Murthy, V.S.S.N.V.G. "Soret and Dufour effects on doublediffusive free convection from a corrugated vertical surface in a non-Darcy porous medium", Trans. Porous Media, 85, pp. 117-130 (2010).

23. Hayat, T., Shehzad, S.A., Qasim, M. and Obaidat, S. "Flow of a second grade fluid with convective boundary conditions", Therm. Sci., 15(2), S253-S261 (2011).

24. Makinde, O.D. and Aziz, A. "MHD mixed convection from a vertical plate embedded in a porous medium with a convective boundary condition", Int. J. Therm. Sci., 49, pp. 1813-1820 (2010).

25. Shehzad, S.A., Alsaedi, A. and Hayat, T. "Threedimensional flow of Jeffery fluid with convective surface boundary conditions", Int. J. Heat Mass Transf., 55, pp. 3971-3976 (2012).

26. Makinde, O.D. "Similarity solution for natural convection from a moving vertical plate with internal heat generation and a convective boundary condition", Therm. Sci., 15(1), pp. S137-S143 (2011).

27. Hayat, T., Waqas, M., Shehzad, S.A. and Alsaedi, A. "Effects of Joule heating and thermophoresis on stretched flow with convective boundary conditions", Scientia Iranica B, 21(3), pp. 682-692 (2014).

28. Liao, S.J., Beyond Perturbation: Introduction to Homotopy Analysis Method, Chapman and Hall, CRC, Press, Boca Raton (2003).

29. Hayat, T., Hussain, T., Shehzad, S.A. and Alsaedi, A. "Thermal and concentration stratifications effects in radiative flow of Jeffrey fluid over a stretching sheet", Plos One, 9(10), pp. 1-15 (2014).

\section{Biographies}

Sheniyappan Eswaramoorthi is an Assistant Professor in the Department of Mathematics, Dr. N.G.P. Arts and Science College, Coimbatore, Tamilnadu, India. He obtained his MSc degree in Mathematics from Erode Arts and Science College, Erode, India, and MPhil degree from Bharathiar University, Coimbatore, India. He is currently doing $\mathrm{PhD}$ degree in the field of boundary layer flow, heat and mass transfer of a viscoelastic fluid.

Marimuthu Bhuvaneswari received MSc, MPhil, and $\mathrm{PhD}$ degrees from Bharathiar University, Coimbatore, India. She received the Post-Doctoral Fellowship twice from National Cheng Kung University, 
Taiwan, and twice from Sungkyunkwan University, South Korea. Then, she worked as a research fellow in University of Malaya, Malaysia. Her areas of interest are numerical and analytical methods for PDE, radiation, and heat and mass transfer.

Sivanandam Sivasankaran received his MSc, MPhil, and $\mathrm{PhD}$ degrees from Bharathiar University, India, in 2000, 2002, 2006, respectively. Afterwards, he received Post-Doctoral Fellowship from National Cheng Kung University, Taiwan, and National Taiwan University, Taiwan. He was a Research Professor in Yonsei University, South Korea. He was an Assistant Professor in Sungkyunkwan University, South Korea. Presently, he is a Senior Lecturer in the Institute of Mathematical Sciences, University of Malaya, Malaysia. $\mathrm{He}$ is a member of editorial board in several international journals and reviewer of more than 20 international journals. He is an Associate Editor in Journal of Applied Fluid Mechanics \& Frontiers in Mechanical Engineering. His areas of interest are convective heat and mass transfer, CFD, nanofluids, micro-channel heat sinks, and porous media.

Subbarayagounder Rajan is an Associate Professor of Mathematics at Erode Arts and Science College, Erode, Tamilnadu, India, having 25 years of teaching experience at both undergraduate and postgraduate levels. He received his MSc degree from Madras University in 1984, and MPhil and PhD degrees from Bharathiar University, India, in 1995 and 2005, respectively. His areas of interest are computer algebra systems and applications of differential equations. 